\title{
Absolute Reference Values of the Real Gas
}

\author{
Albrecht Elsner \\ Am Mühlbach 14, D-85748 Garching, Germany \\ Email: alimeli.elsner@gmail.com
}

How to cite this paper: Elsner, A. (2018) Absolute Reference Values of the Real Gas. Engineering, 10, 270-290.

https://doi.org/10.4236/eng.2018.105019

Received: April 2, 2018

Accepted: May 22, 2018

Published: May 25, 2018

Copyright $\odot 2018$ by author and Scientific Research Publishing Inc. This work is licensed under the Creative Commons Attribution International License (CC BY 4.0).

http://creativecommons.org/licenses/by/4.0/

\begin{abstract}
With his publication in 1873 [1] J. W. Gibbs formulated the thermodynamic theory. It describes almost all macroscopically observed properties of matter and could also describe all phenomena if only the free energy $U-S T$ were explicitly known numerically. The thermodynamic uniqueness of the free energy obviously depends on that of the internal energy $U$ and the entropy $S$, which in both cases Gibbs had been unable to specify. This uncertainty, lasting more than 100 years, was not eliminated either by Nernst's hypothesis $S=0$ at $T=0$. This was not achieved till the advent of additional proof of the thermodynamic relation $U=0$ at $T=T_{c}$. It is noteworthy that from purely thermodynamic consideration of intensive and extensive quantities it is possible to derive both Gibbs's formulations of entropy and internal energy and their now established absolute reference values. Further proofs of the vanishing value of the internal energy at the critical point emanate from the fact that in the case of the saturated fluid both the internal energy and its phase-specific components can be represented as functions of the evaporation energy. Combining the differential expressions in Gibbs's equation for the internal energy, $\mathrm{d}(\mu / T) / \mathrm{d}(1 / T)$ and $\mathrm{d}(p / T) / \mathrm{d}(1 / T)$, to a new variable $\mathrm{d}(\mu / T) / \mathrm{d}(p / T)$ leads to a volume equation with the lower limit $V_{c}$ as boundary condition. By means of a variable transformation one obtains a functional equation for the sum of two dimensionless variables, each of them being related to an identical form of local interaction forces between fluid particles, but the different particle densities in the vapor and liquid spaces produce different interaction effects. The same functional equation also appears in another context relating to the internal energy. The solution of this equation can be given in analytic form and has been published [2] [3]. Using the solutions emerging in different sets of problems, one can calculate absolutely the internal energy as a function of temperature-dependent, phase-specific volumes and vapor pressure.
\end{abstract}

\section{Keywords}

Entropy Reference Value $S(M, V, 0)=0$, Internal Energy Reference Value 
$U\left(M, V, T_{c}\right)=0$, Critical Volume $V=M \cdot[\mathrm{d}(\mu / T) / \mathrm{d}(p / T)]_{c}$, Two-Phase

Equilibrium $-M\left(u_{v}-u_{l}\right)(0) \leq S T+M \mu=M\left[v_{v}+v_{l}-\left(v_{v}-v_{l}\right) / \ln \left(v_{v} / v_{l}\right)\right]$

$\cdot \mathrm{d}(p / T) / \mathrm{d}(1 / T)+V \mathrm{~d} p / \mathrm{d} T \cdot T=U+V p \leq V p \leq V p_{c}$, Chemical Potential

Negative, $M \mu(T) \leq M \mu(0)=U(M, V, 0)=M \cdot\left[-\left(u_{v}-u_{l}\right)(0)\right]<0$.

\section{Introduction}

The purpose of this paper is to show that Gibbs's theory [1] contains intrinsically "natural" reference values of entropy and internal energy that are reciprocal and thus represent thermodynamic reference values [4]. Nevertheless, it was and is still accepted till the recent past that the value of the internal energy of the real gas cannot be given absolutely, e.g. [5] [6]. Instead, the calculation of entropy and internal energy could be based on a so-called fiducial reference value [7]. To put an end to the diversity of individually, arbitrarily chosen fiducial values, it was decided at conferences in the 1950s to assign the values of entropy and internal energy (or enthalpy) of a liquid at the triple point the reference values zero, which in any case the Nernst hypothesis contradicts. On this basis mathematically complex equations were put forward, but an analysis (see Appendix) shows that they contain thermodynamic inconsistencies. These then yielded incorrect thermodynamic data published for a large number of gases, e.g. [8]-[15]. None of these skeleton tables presents data on the chemical potential, which could have been derived from the differences $h_{v, l}-s_{v, l} T$. A paper that specifically investigates calculation of the chemical potential of the generally accepted conference agreements comes to the surprising conclusion that the chemical potential increases as the temperature [16]. This result cannot, however, account for daily observation that flow of freely-moving matter occurs from cold to warm regions [17]. One has to take the consequence from the numerous thermodynamic discrepancies ensuing from calculating entropy and internal energy from the assumptions mentioned, $s_{l}\left(T_{t}\right)=0$ and $u_{l}\left(T_{t}\right)=0$ (or $\left.h_{l}\left(T_{t}\right)=0\right)$. Here, too, as so often in the history of physics, one has to abandon a trusted hypothesis. Here it is the assumption of the naive addition of fiducial constants to thermodynamic fundamental quantities.

The paper shows four possible ways of finding directly the thermodynamic reference values of entropy and internal energy and thus comply with the correct treatment of real properties of matter called for by Gibbs. First there is the possibility of studying the characteristic features of intensive and extensive quantities in order to describe thermodynamically the real properties of matter. A second investigation deals with the question what follows from representation of the internal energy of the fluid as a function of the evaporation energy. The third possibility is concerned with solution of a functional equation for the sum of two dimensionless variables, the one referring to the local interaction potential of fluid particles in the vapor space and the other to that in the liquid. 
The functional equation emerges from the original equation for the internal energy and has been solved [3]. The functional equation is, on the other hand, also encountered when one represents the particular phase-specific internal energy as a funtion of the evaporation or condensation energy. It has of course the same (physically unique) solution and allows the internal energy to be explicitly calculated as an absolutely determined temperature function of the measurable quantities: phase-specific volumes and vapor pressure [2].

\section{Intensive and Extensive Quantities of the Saturated Fluid}

The homogeneity of the fluid allows its macroscopic properties to be described by intensive and extensive quantities.

The intensive quantities are the temperature $T$, vapor pressure $p$ and chemical potential $\mu$; vapor pressure and chemical potential are pure temperature functions below the critical point of the fluid. With the finite critical values $p_{c}$ and $\mathrm{d}^{n-1} p / \mathrm{d} T_{c}^{n-1}>\mathrm{d}^{n} p / \mathrm{d} T_{c}^{n} \quad(n=1,2,3, \cdots)$ the $\epsilon$-expansion of $p$ to second order yields

$$
\begin{gathered}
0 \leq T \leq T_{c}, \quad 0 \leq \epsilon=1-T / T_{c} \leq 1, \\
0 \leq p=p_{c}-\frac{\mathrm{d} p}{\mathrm{~d} T_{\mid c}} T_{c} \epsilon+\frac{1}{2} \frac{\mathrm{d}^{2} p}{\mathrm{~d} T^{2}{ }_{\mid c}} T_{c}^{2} \epsilon^{2} \leq p_{c}, \\
0 \leq \frac{\mathrm{d} p}{\mathrm{~d} T}=\frac{\mathrm{d} p}{\mathrm{~d} T_{\mid c}}-\frac{\mathrm{d}^{2} p}{\mathrm{~d} T^{2}{ }_{\mid c}} T_{c} \epsilon+\frac{1}{2} \frac{\mathrm{d}^{3} p}{\mathrm{~d}^{3}{ }_{\mid c}} T_{c}^{2} \epsilon^{2} \leq \frac{\mathrm{d} p}{\mathrm{~d} T_{\mid c}}, \\
0 \leq \frac{\mathrm{d}^{2} p}{\mathrm{~d} T^{2}}=\frac{\mathrm{d}^{2} p}{\mathrm{~d} T^{2}{ }_{k}}-\frac{\mathrm{d}^{3} p}{\mathrm{~d} T^{3}{ }_{\mid c}} T_{c} \epsilon+\cdots \leq \frac{\mathrm{d}^{2} p}{\mathrm{~d} T^{2}{ }_{\mid c}}, \\
\frac{\mathrm{d}(p / T)}{\mathrm{d}(1 / T)}=p-\frac{\mathrm{d} p}{\mathrm{~d} T} T \leq 0, \quad \frac{\mathrm{d}}{\mathrm{d} T} \frac{\mathrm{d}(p / T)}{\mathrm{d}(1 / T)}=-\frac{\mathrm{d}^{2} p}{\mathrm{~d} T^{2}} T \leq 0 .
\end{gathered}
$$

For $T \geq 0$ the vapor pressure is a positive and convexly curved temperature function increasing with $T$ from 0 to $p_{c}$. Also the temperature derivatives increase with $T$ from $\mathrm{d}^{n} p / \mathrm{d} T^{n}(0)=0$ to finite values $\mathrm{d}^{n} p / \mathrm{d} T^{n}\left(T_{c}\right)$ (where $n=1,2, \cdots)$. In contrast, the chemical potential is a negative and concavely curved temperature function decreasing with $T$. With the finite critical values $\mu_{c}$ and $\mathrm{d} \mu / \mathrm{d} T_{c}$, and, on the other hand, the divergent terms $\mathrm{d}^{n} \mu / \mathrm{d} T_{c}^{n}$ $(n=2,3, \cdots)$ an $\epsilon$-expansion of $\mu$ is not possible:

$$
\begin{aligned}
& 0>\mu \geq \mu_{c}, 0 \geq \frac{\mathrm{d} \mu}{\mathrm{d} T} \geq \frac{\mathrm{d} \mu}{\mathrm{d} T_{\mid c}}, 0 \geq \frac{\mathrm{d}^{2} \mu}{\mathrm{d} T^{2}} \geq \frac{\mathrm{d}^{2} \mu}{\mathrm{d} T^{2}{ }_{k}} \rightarrow-\infty, \\
& 0>\frac{\mathrm{d}(\mu / T)}{\mathrm{d}(1 / T)} \geq \frac{\mathrm{d}(\mu / T)}{\mathrm{d}(1 / T)_{\mid c}}, 0 \leq \frac{\mathrm{d}}{\mathrm{d} T} \frac{\mathrm{d}(\mu / T)}{\mathrm{d}(1 / T)}=-\frac{\mathrm{d}^{2} \mu}{\mathrm{d} T^{2}} T<-\frac{\mathrm{d}^{2} \mu}{\mathrm{d} T^{2}{ }_{\mid c}} T_{c} \rightarrow+\infty, \\
& 0>\frac{\mu}{p}>\frac{\mu}{p_{\mid c}}, 0>\frac{\mathrm{d} \mu}{\mathrm{d} p}>\frac{\mathrm{d} \mu}{\mathrm{d} p_{\mid c}}, 0<\frac{\mathrm{d}^{2} \mu}{\mathrm{d}^{2}{ }_{\mid c}}<\frac{\mathrm{d}^{2} \mu}{\mathrm{d} p^{2}}, 0<\frac{\mathrm{d}(\mu / T)}{\mathrm{d}(p / T)_{\mid c}} \leq \frac{\mathrm{d}(\mu / T)}{\mathrm{d}(p / T)} .
\end{aligned}
$$

Thermodynamics treats the quantity $X$ as an extensive quantity, which means that $X$ is proportional to the fluid mass $M$. The validity of the relation $X=x M$ leads to $x$ having the property of additivity and ensures its uniqueness. At 
temperatures below the critical point the fluid mass $M$ in the volume $V$ is additively composed of the vapor mass $M_{V}$ and the condensed mass $M_{l}$ in the sub-volumes $V_{v}$ and $V_{i}: M=M_{v}+M_{l}$ in $V=V_{v}+V_{l}$. The same applies to other extensive quantities such as the entropy $S$, internal energy $U$, enthalpy $H$, free energy $F$, and heat capacity $C$. Denoting such quantities by $X$ and $X_{v, l}$ and the corresponding mass-specific quantities by $x=X / M$ and $x_{v, l}=X_{v, l} / M_{v, l}$, one obtains the following definitions:

$$
\begin{aligned}
& M=M_{v}+M_{l}, \quad X=X_{v}+X_{l}, \quad X=\{V, S, U, H, F, C\}, \\
& x=X / M, \quad x_{v, l}=X_{v, l} / M_{v, l}, \quad x=\{v, s, u, h, f, c\} .
\end{aligned}
$$

It is worth mentioning that the temperature variation of the ratio of the differences $\left(x-x_{l}\right)$ and $\left(x_{v}-x\right)$ of quantities such as the volume, entropy, internal energy, enthalpy, free energy, and specific heat is the same and equal to that of the ratio of vapor to condensed masses, viz.

$$
0 \leq \frac{M_{v}}{M_{l}}=\frac{v-v_{l}}{v_{v}-v}=\frac{s-s_{l}}{s_{v}-s}=\frac{u-u_{l}}{u_{v}-u}=\frac{h-h_{l}}{h_{v}-h}=\frac{f-f_{l}}{f_{v}-f}=\frac{c-c_{l}}{c_{v}-c} \leq 1,
$$

where the equality signs are valid for $T=0$ and $T=T_{c}$, respectively. From Equation (1.4) one arrives at the interdependence of two extensive quantities $X$ and $y$ and their phase-specific values $x_{v, l}$ and $y_{v, l}$ in the following form:

$$
x\left(y_{l}-y_{v}\right)+y\left(x_{v}-x_{l}\right)-x_{v} y_{l}+x_{l} y_{v}=0 \text {. }
$$

This relationship can also be deduced from the correlations $x=\left(M_{v} / M\right) \cdot x_{v}+\left(M_{l} / M\right) \cdot x_{l} \quad$ and $\quad y=\left(M_{v} / M\right) \cdot y_{v}+\left(M_{l} / M\right) \cdot y_{l} \quad$ with $\left(M_{v} / M\right)+\left(M_{l} / M\right)=1$.

The decomposition of mass $M$ into $M_{v}$ and $M_{l}$ below the critical point occurs within limits and is given by

$$
0 \leq \frac{M_{v}}{M}=\frac{v-v_{l}}{v_{v}-v_{l}} \leq \frac{1}{2} \leq \frac{M_{l}}{M}=\frac{v_{v}-v}{v_{v}-v_{l}} \leq 1 .
$$

While the mass-specific quantity $x$ constitutes an average of the quantity $X$ in $V$ in relation to the total mass $M$ and is thus a function of $T$ and $V / M$, the massand phase-specific quantity $X_{V}$ describes the quantity $X_{V}$ in the volume $V_{V}$ and is related to the vapor mass $M_{v}$, and the quantity $x_{l}$ describes $X_{l}$ in $V_{l}$ and is related to $M_{l}$. The quantities $x_{v, l}$ then give thermodynamic information on the masses $M_{v, l}$ in $V_{v, l}$, which are subject to equal values of temperature $T$, vapor pressure $p=p(T)$ and chemical potential $\mu=\mu(T)$ in $V$, and different density values $v_{v}^{-1}$ in $V_{v}$ and $v_{l}^{-1}$ in $V_{l}$. Since the densities of the vapor and condensate in $V_{v}$ and $V_{l}$ are functions of the temperature alone, the quantities $x_{v, l}$ in $V_{v, l}$ are likewise pure temperature functions. As $V_{V}$ is different to $V_{l}$ for $T<T_{c}$, the value $x_{V}$ is different to $x_{l}$ for $T<T_{c}$, and as $V_{V}$ and $v_{l}$ are equal to the critical value $V_{c}$ at the critical point $\left(T_{c}, p_{c}, \mu_{c}\right)$, the values $x_{V}$ and $x_{l}$ are equal to the critical value $x_{c}$ at $\left(T_{c}, v_{c}\right)$. The information from $x_{V}$ and $x_{l}$ implicitly contains all particle interactions that can be expressed in terms of various imaginable types of descriptions of thermodynamic properties. 
If a thermodynamic quantity is represented in its domain of definition $[T, v]$ by a thermodynamic function $x(T, v)$, a thermodynamic quantity is always an absolute quantity. For example, the physics of the real gas operates in the temperature range $\left[0, T_{c}\right]$ in the limits $0=S(0, M, V)<S(T, M, V)<S\left(T_{c}, M, V\right)=\left[V p_{c}-M \mu_{c}\right] / T_{c}$ and $-M\left(u_{v}-u_{l}\right)(0)=U(0, M, V)<U(T, M, V)<U\left(T_{c}, M, V\right)=0$.

The critical value $x_{c}$ of the quantity $x=\{v, s, u, f\}$ is finite. In fact, when $x_{v}$ approaches the finite value $x_{c}$ from below, then $x_{l}$ approaches $x_{c}$ from above and vice versa; in any case, one has $x_{v}\left(T_{c}\right)-x_{l}\left(T_{c}\right)=0$. The approach is determined by $\lim _{T \rightarrow T_{c}}\left(\mathrm{~d} x_{v}(T) / \mathrm{d} T\right)=-\lim _{T \rightarrow T_{c}}\left(\mathrm{~d} x_{l}(T) / \mathrm{d} T\right)$. The ratio value of $x_{v}(T) / x_{l}(T)$ at the critical point is then different for the two possible cases of equal or opposite sign of $x_{v}(T)$ and $x_{l}(T)$. One has

$$
\begin{array}{ll}
x_{v}\left(T_{c}\right)=x_{l}\left(T_{c}\right) \neq 0, x_{v}\left(T_{c}\right) / x_{l}\left(T_{c}\right)=+1 & \text { for } x_{v}(T) / x_{l}(T)>0, \\
x_{v}\left(T_{c}\right)=x_{l}\left(T_{c}\right)=0, x_{v}\left(T_{c}\right) / x_{l}\left(T_{c}\right)=-1 & \text { for } x_{v}(T) / x_{l}(T)<0 .
\end{array}
$$

The relations of the first line of (1.7) are valid for the quantities $x=\{v, s, f\}$.

The consequence from relations (1.7) for the quantity $x=u$ shall be investigated. It can be stated that the vapor energy $U_{v}$ at low temperatures is positive since vapor particles are so far apart that their (negative) interaction potentials are vanishingly small in comparison with their (positive) thermal energies. At low temperatures one thus has $u_{v}=U_{v} / M_{v}>0$. Under these conditions the evaporation energy $\left(u_{v}-u_{l}\right) M$ is very much larger than $U_{v}$. From $\left(u_{v}-u_{l}\right) M \gg U_{v}$ one obtains $\left(u_{v}-u_{l}\right) \gg U_{v} / M=u_{v} M_{v} / M$ or, with $M_{v} / M \leq 1 / 2,\left(u_{v}-u_{l}\right)>u_{v}>u_{v} / 2>u_{v} M_{v} / M \geq 0$. This gives an estimate of the mass-specific energy $u_{l}$ in the form of $u_{l}=u_{v}-\left(u_{v}-u_{l}\right) \leq 0$, i.e. the vapor energy $u_{v}$ is not negative and the condensate energy $u_{l}$ is not positive. The second line of relations (1.7) then states $u_{c}=0$. This yields the important results,

$$
u_{l}(T) \leq 0 \leq u_{v}(T), u_{l}\left(T_{c}\right)=0=u_{v}\left(T_{c}\right), u_{v}\left(T_{c}\right) / u_{l}\left(T_{c}\right)=-1 .
$$

From Equations (1.3) one obtains the thermodynamic relation of the mean fluid quantity $x$ (which is a function of $T$ and $v$ ) to the phase-specific quantities $x_{v, l}$ (which are pure temperature functions) as follows:

$$
\begin{aligned}
x(T, v) & =\frac{M_{v}}{M}(T, v) x_{v}(T)+\frac{M_{l}}{M}(T, v) x_{l}(T)=x_{v}-\frac{M_{l}}{M}\left(x_{v}-x_{l}\right) \\
& =x_{l}+\frac{M_{v}}{M}\left(x_{v}-x_{l}\right)=\frac{v-v_{l}}{v_{v}-v_{l}} x_{v}+\frac{v_{v}-v}{v_{v}-v_{l}} x_{l} \\
& =x_{v}-\frac{v_{v}-v}{v_{v}-v_{l}}\left(x_{v}-x_{l}\right)=x_{l}+\frac{v-v_{l}}{v_{v}-v_{l}}\left(x_{v}-x_{l}\right) .
\end{aligned}
$$

Differentiation of $x(T, v)$ with respect to $v$ at fixed $T$ yields the coefficient of isothermal phase transition,

$$
\left(\frac{\partial x}{\partial v}\right)_{T}=\frac{x_{v}-x_{l}}{v_{v}-v_{l}} .
$$


Thus the fluid quantity $x\left(T, v_{c}\right)$ can be expressed in terms of $x_{v, l}, v_{v, l}, v_{c}$, and $(\partial x / \partial v)_{T}$ :

$$
x\left(T, v_{c}\right)=x_{v, l}(T)-\left(v_{v, l}(T)-v_{c}\right)(\partial x / \partial v)_{T} .
$$

If the saturated fluid does not have the critical volume $v_{\mathcal{c}}$ but the volume $v$, the following conversion has to be made:

$$
x(T, v)=x\left(T, v_{c}\right)-\left(v_{c}-v\right)(\partial x / \partial v)_{T}=x_{v, l}-\left(v_{v, l}-v\right)(\partial x / \partial v)_{T} .
$$

At $T=T_{c}$ the fluid takes the critical volume $v_{c}$. From Equation (1.9) it is immediately obvious that the critical values $x\left(T_{c}, v_{c}\right), x_{v}\left(T_{c}\right), x_{l}\left(T_{c}\right)$, and the zero-point values $x(0, v)$ and $x_{l}(0)$ are respectively equal,

$$
x\left(T_{c}, v_{c}\right)=x_{v}\left(T_{c}\right)=x_{l}\left(T_{c}\right)=x_{c}, \quad x(0, v)=x_{l}(0),
$$

and from Equation (1.10) it follows that

$$
\begin{array}{ll}
x_{l}(T)<x(T, v)<x_{v}(T) & \text { if }(\partial x / \partial v)_{T}>0, \\
x_{l}(T)>x(T, v)>x_{v}(T) & \text { if }(\partial x / \partial v)_{T}<0 .
\end{array}
$$

In the theory it is not only the difference of the phase-specific quantities $X_{V}$ and $x_{p}$ i.e.

$$
x_{v}-x_{l}=\left(v_{v}-v_{l}\right)(\partial x(T, v) / \partial v)_{T},
$$

that is of importance, but also their sum

$\left(x_{v}+x_{l}\right)=2 x(T, v)+\left(v_{v}+v_{l}-2 v\right)(\partial x(T, v) / \partial v)_{T}$. Since

$\left(v_{v}+v_{l}-2 v\right) /\left(v_{v}-v_{l}\right)=\left(\left[v_{v}-v\right]-\left[v-v_{l}\right]\right) /\left(v_{v}-v_{l}\right)=M_{l} / M-M_{v} / M \geq 0$, one has

$$
x_{v}+x_{l}=2 x+\left(x_{v}-x_{l}\right)\left(M_{l} / M-M_{v} / M\right) .
$$

The sign of the function $\left(x_{v}+x_{l}\right)$ will subsequently be of interest. It is the same as that of the function $\left(x_{v}-x_{l}\right)$ if the product function $\left(x_{v}+x_{l}\right)\left(x_{v}-x_{l}\right)$ has a positive sign, while the signs of $\left(x_{v}+x_{l}\right)$ and $\left(x_{v}-x_{l}\right)$ are opposite if

$\left(x_{v}+x_{l}\right)\left(x_{v}-x_{l}\right)=2 x\left(x_{v}-x_{l}\right)+\left(x_{v}-x_{l}\right)^{2}\left(M_{l} / M-M_{v} / M\right)$ is negative. The latter can be the case if either the product function $x\left(x_{v}-x_{l}\right)$ or the difference $\left(x_{v}^{2}-x_{l}^{2}\right)$ is negative (see Equations (1.25) and (1.26) below). At the critical point one has $\left(x_{v}+x_{l}\right)\left(T_{c}\right)=2 x\left(T_{c}, v_{c}\right)$ and at absolute zero $\left(x_{v}+x_{l}\right)(0)=2 x(0, v)+\left(x_{v}-x_{l}\right)(0)$ and $x_{v}(0)=x(0, v)+\left(x_{v}-x_{l}\right)(0)$; for the vapor-phase quantity $x_{v}$ one then obtains

$$
x_{v}\left(T_{c}\right)=2 x\left(T_{c}, v_{c}\right)-x_{l}\left(T_{c}\right), \quad x_{v}(0)=x(0, v)+\left(x_{v}-x_{l}\right)(0) .
$$

The critical mean fluid value $x\left(T_{c}, v_{c}\right)$ is thus equal to the mean of the phase-specific critical values $\frac{1}{2}\left[x_{v}\left(T_{c}\right)+x_{l}\left(T_{c}\right)\right]$ and, if the fluid value $x(0, v)$ at absolute zero is given by the condensation energy value $-\left(x_{v}-x_{l}\right)(0)$, the vapor value $x_{v}(0)$ vanishes, i.e. it holds, for example, that $u_{v}(0)=0$. 
At this place the quantum state of the Bose-Einstein condensation should be noticed. In contrast to the thermodynamic temperature absolute zero the lowest temperature available is the transition temperature $T_{t r}=\left(\hbar^{2} / m k\right) \cdot\left(2 \pi / \mathrm{e}^{5 / 3}\right) \cdot n^{2 / 3}>0$, where $\hbar=h / 2 \pi$ is the Planck constant, $k$ the Boltzmann constant, $m$ the particle mass and $n$ the particle density in the condensate. The atomic densities achieved in experiments range from $10^{-14}$ to $10^{-15} \mathrm{~cm}^{-3}$ and transition temperatures from $100 \mathrm{nK}$ to a few $\mu \mathrm{K}$ [18]. The internal energy of the dilute gas is positive for $T>T_{t r}$ and vanishes at $T=T_{t r}$, whereas that of the condensate is negative [19].

\subsection{Interdependence of Extensive and Intensive Quantities}

Since the two-phase equilibrium can be described by extensive as well by intensive quantities, an interdependence between these quantities exists. Thermodynamics yields for the quotients $\left(x_{v}-x_{l}\right) /\left(v_{v}-v_{l}\right)$ of the particular quantities $x=\{f, s, u, c\}$ the well-known vapor pressure relations,

$$
\begin{aligned}
& \left(\frac{\partial f}{\partial v}\right)_{T}=\frac{f_{v}-f_{l}}{v_{v}-v_{l}}=-p \leq 0,\left(\frac{\partial s}{\partial v}\right)_{T}=\frac{s_{v}-s_{l}}{v_{v}-v_{l}}=\frac{\mathrm{d} p}{\mathrm{~d} T} \geq 0, \\
& \left(\frac{\partial u}{\partial v}\right)_{T}=\frac{u_{v}-u_{l}}{v_{v}-v_{l}}=-\frac{\mathrm{d}(p / T)}{\mathrm{d}(1 / T)} \geq 0,\left(\frac{\partial c}{\partial v}\right)_{T}=\frac{c_{v}-c_{l}}{v_{v}-v_{l}}=\frac{\mathrm{d}^{2} p}{\mathrm{~d} T^{2}} T \geq 0 .
\end{aligned}
$$

Correspondingly, for the quotients $\left(x_{v} v_{l}-x_{l} v_{v}\right) /\left(v_{v}-v_{l}\right)$ the chemical potential relations are

$$
\begin{gathered}
\frac{f_{v} v_{l}-f_{l} v_{v}}{v_{v}-v_{l}}=-\mu>0, \quad \frac{s_{v} v_{l}-s_{l} v_{v}}{v_{v}-v_{l}}=\frac{\mathrm{d} \mu}{\mathrm{d} T} \leq 0, \\
\frac{u_{v} v_{l}-u_{l} v_{v}}{v_{v}-v_{l}}=-\frac{\mathrm{d}(\mu / T)}{\mathrm{d}(1 / T)}>0, \quad \frac{c_{v} v_{l}-c_{l} v_{v}}{v_{v}-v_{l}}=\frac{\mathrm{d}^{2} \mu}{\mathrm{d} T^{2}} T \leq 0 .
\end{gathered}
$$

Equations (1.18) and (1.19) allow one to define volume functions $\left(x_{v} v_{l}-x_{l} v_{v}\right) /\left(x_{v}-x_{l}\right)$, which can be represented in different ways:

$$
\begin{aligned}
\frac{x_{v} v_{l}-x_{l} v_{v}}{x_{v}-x_{l}} & =v_{v}+v_{l}-\frac{x_{v} v_{v}-x_{l} v_{l}}{x_{v}-x_{l}}=v_{l}+\frac{v_{v}-v_{l}}{1-x_{v} / x_{l}} \\
& =v_{v}-\frac{v_{v}-v_{l}}{1-x_{l} / x_{v}}=\frac{v_{v}+v_{l}}{2}-\frac{v_{v}-v_{l}}{2} \frac{x_{v}+x_{l}}{x_{v}-x_{l}} .
\end{aligned}
$$

In evaluating the critical value of a volume function (1.20) one should give heed, in respect of Equations (1.7) in the case $x_{v}\left(T_{c}\right) / x_{l}\left(T_{c}\right)=1$, to whether a finite limiting value exists; in the case $x_{v}\left(T_{c}\right) / x_{l}\left(T_{c}\right)=-1$ the limiting value is $v_{c}$. Hence the result is:

$$
\frac{u_{v} v_{l}-u_{l} v_{v}}{u_{v}-u_{l}}\left(T_{c}\right)=\frac{\mathrm{d}(\mu / T)}{\mathrm{d}(p / T)}\left(T_{c}\right)=v_{c} .
$$

With $x_{v, l}=\left\{f_{v, l}, s_{v, l}, u_{v, l}, c_{v, l}\right\}$ one obtains

$$
\frac{\mu}{p}=\frac{f_{v} v_{l}-f_{l} v_{v}}{f_{v}-f_{l}}<0, \quad \frac{\mathrm{d} \mu}{\mathrm{d} p}=\frac{s_{v} v_{l}-s_{l} v_{v}}{s_{v}-s_{l}}<0,
$$




$$
\frac{\mathrm{d}(\mu / T)}{\mathrm{d}(p / T)}=\frac{u_{v} v_{l}-u_{l} v_{v}}{u_{v}-u_{l}}>0, \quad \frac{\mathrm{d}^{2} \mu}{\mathrm{d} T^{2}} / \frac{\mathrm{d}^{2} p}{\mathrm{~d} T^{2}}=\frac{c_{v} v_{l}-c_{l} v_{v}}{c_{v}-c_{l}}<0 .
$$

From Equations (1.22) it immediately follows that the critical value is finite for $x=\{f, s, u\}$ and divergent for $x=c$.

\subsection{Entropy and Internal Energy Relations}

According to relation (1.5), the interdependence of volume and entropy is $v\left(s_{l}-s_{v}\right)+s\left(v_{v}-v_{l}\right)-v_{v} s_{l}+v_{l} s_{v}=0$. Taking relations (1.18) and (1.19) into account, viz. $\left(s_{v}-s_{l}\right)=\left(v_{v}-v_{l}\right) \cdot \mathrm{d} p / \mathrm{d} T$ and $\left(v_{v} s_{l}-v_{l} s_{v}\right) /\left(v_{v}-v_{l}\right)=-\mathrm{d} \mu / \mathrm{d} T$, this can be transformed to $0 \leq-\frac{\mathrm{d} \mu}{\mathrm{d} T}=\frac{s_{l} v_{v}-v_{l} s_{v}}{v_{v}-v_{1}}=s-v \frac{\mathrm{d} p}{\mathrm{~d} T}$. Thus one gets Gibbs's entropy relations, including the thermodynamic reference value 0 :

$$
\begin{gathered}
0 \leq v \frac{\mathrm{d} p}{\mathrm{~d} T} \leq-\frac{\mathrm{d} \mu}{\mathrm{d} T}+v \frac{\mathrm{d} p}{\mathrm{~d} T}=s(v, T), \\
0 \leq s_{l}(T), \quad s_{v, l}(T)=-\frac{\mathrm{d} \mu}{\mathrm{d} T}+v_{v, l} \frac{\mathrm{d} p}{\mathrm{~d} T}=s(v, T)+\left[v_{v, l}(T)-v\right] \frac{\mathrm{d} p}{\mathrm{~d} T} .
\end{gathered}
$$

The value $s(v, T)$ is the sum of the terms $v \cdot \mathrm{d} p / \mathrm{d} T \geq 0$ and $(-\mathrm{d} \mu / \mathrm{d} T) \geq 0$ and is positive for $T>0$ and vanishes for $T=0$. The same is valid for the phase-specific entropy $s_{l}(T)$; and since $0<v_{l}(T) \leq v\left(T_{c}\right)=v_{c} \leq v_{v}(T)$ one obtains the following sequences

$$
0 \leq s_{l}(T) \leq s\left(v_{c}, T\right) \leq s\left(v_{c}, T_{c}\right)=s_{c} \leq s_{v}(T) .
$$

The interdependence of volume and internal energy is $v\left(u_{l}-u_{v}\right)+u\left(v_{v}-v_{l}\right)-v_{v} u_{l}+v_{l} u_{v}=0$, which with $\left(u_{v}-u_{l}\right)=-\left(v_{v}-v_{l}\right) \cdot \mathrm{d}(p / T) / \mathrm{d}(1 / T)$ and $\left(v_{v} u_{l}-v_{l} u_{v}\right) /\left(v_{v}-v_{l}\right)=-\mathrm{d}(\mu / T) / \mathrm{d}(1 / T)$ gives Gibbs's internal energy relations and with respect to relation (1.21) the thermodynamic reference value 0 :

$$
\begin{aligned}
& u(v, T)=\frac{\mathrm{d}(\mu / T)}{\mathrm{d}(1 / T)}-v \frac{\mathrm{d}(p / T)}{\mathrm{d}(1 / T)} \leq \frac{\mathrm{d}(\mu / T)}{\mathrm{d}(1 / T)}\left(T_{c}\right)-v_{c} \frac{\mathrm{d}(p / T)}{\mathrm{d}(1 / T)}\left(T_{c}\right) \leq 0, \\
& u_{v, l}(T)=\frac{\mathrm{d}(\mu / T)}{\mathrm{d}(1 / T)}-v_{v, l}(T) \frac{\mathrm{d}(p / T)}{\mathrm{d}(1 / T)}=u(v, T)-\left[v_{v, l}(T)-v\right] \frac{\mathrm{d}(p / T)}{\mathrm{d}(1 / T)} .
\end{aligned}
$$

For $0 \leq T \leq T_{c}$ the value $u(v, T)$ is the sum of the negative term $\mathrm{d}(\mu / T) / \mathrm{d}(1 / T)$ and the positive term $(-v \cdot \mathrm{d}(p / T) / \mathrm{d}(1 / T))$, where it holds that $\mathrm{d}(\mu / T) / \mathrm{d}(1 / T)-v \cdot \mathrm{d}(p / T) / \mathrm{d}(1 / T) \leq 0$ and therefore $u(v, T)$ is not positive. Furthermore, the phase-specific internal energies obey the relations $\left(u_{v}-u_{l}\right)=-\left(v_{v}-v_{l}\right) \mathrm{d}(p / T) / \mathrm{d}(1 / T) \geq 0$ and $\left(u_{v}+u_{l}\right) \leq 0$; the last relation follows from $\left(u_{v}+u_{l}\right)\left(u_{v}-u_{l}\right)=\left(u_{v}^{2}-u_{l}^{2}\right)=-2\left(v_{v}-v_{l}\right)[\mathrm{d}(p / T) / \mathrm{d}(1 / T)]^{2}$ $\cdot\left[\mathrm{d}(\mu / T) / \mathrm{d}(p / T)-\left(v_{v}+v_{l}\right) / 2\right] \leq 0 \quad$ since $\quad \mathrm{d}(\mu / T) / \mathrm{d}(p / T)-\left(v_{v}+v_{l}\right) / 2 \geq 0$. Hence one gets

$$
\begin{gathered}
\left(u_{v}-u_{l}\right) \geq 0 \text { and }\left(u_{v}+u_{l}\right) \leq 0, \\
-\left(u_{v}-u_{l}\right) \leq u_{l} \leq u \leq\left(u_{v}+u_{l}\right) \leq u_{c}=0 \leq u_{v} \leq\left(u_{v}-u_{l}\right) .
\end{gathered}
$$

The relations $\left(u_{v}+u_{l}\right)=2 u_{v}-\left(u_{v}-u_{l}\right) \leq 0$ and 
$-\left(u_{v}+u_{l}\right)=-2 u_{l}-\left(u_{v}-u_{l}\right) \geq 0$ lead to the limits of the energies $u_{v, l}$ in relation to the transient energies $\pm\left(u_{v}-u_{l}\right)$ :

$$
0 \leq \frac{u_{v}}{u_{v}-u_{l}} \leq \frac{1}{2} \leq \frac{u_{l}}{-\left(u_{v}-u_{l}\right)} \leq 1 .
$$

In other words: For $0<T<T_{c}$ the vapor internal energy $u_{v}$ is positive and always lower than half the evaporation energy, $u_{v} \leq \frac{1}{2}\left(u_{v}-u_{l}\right)$, and the liquid internal energy $u_{l}$ is negative and lower than half the condensation energy, $u_{l} \leq \frac{1}{2}\left[-\left(u_{v}-u_{l}\right)\right]$. The critical values of the entropy are obtained from the relations $s_{c} T_{c}=v_{c} p_{c}-\mu_{c}=\left(v_{c} \cdot \mathrm{d} p / \mathrm{d} T-\mathrm{d} \mu / \mathrm{d} T\right)_{l c} \cdot T_{c}$, yielding

$$
\begin{aligned}
s\left(v_{c}, T_{c}\right) & =v_{c} \frac{\mathrm{d} p}{\mathrm{~d} T_{k c}}-\frac{\mathrm{d} \mu}{\mathrm{d} T_{k}}=v_{c} \frac{p_{c}}{T_{c}}-\frac{\mu_{c}}{T_{c}} \\
& =\frac{1}{2}\left[v_{c}\left(\frac{p}{T}+\frac{\mathrm{d} p}{\mathrm{~d} T}\right)_{c}-\left(\frac{\mu}{T}+\frac{\mathrm{d} \mu}{\mathrm{d} T}\right)_{c}\right]=-\frac{f_{c}}{T_{c}}>0 .
\end{aligned}
$$

Because $0 \leq c(v, T)=\mathrm{d} u(v, T) / \mathrm{d} T=T \cdot \mathrm{d} s(v, T) / \mathrm{d} T$, the functions $u(v, T)$ and $s(v, T)$ increase monotonically with increasing $T$, and so the critical values present the maximum internal energy and entropy of the saturated fluid.

The interdependence of entropy and internal energy is calculated from the equation $s\left(u_{l}-u_{v}\right)+u\left(s_{v}-s_{l}\right)-s_{v} u_{l}+s_{l} u_{v}=0$ and leads to the following identities and estimates:

$$
s=u \frac{s_{v}-s_{l}}{u_{v}-u_{l}}+\frac{u_{v} s_{l}-u_{l} s_{v}}{u_{v}-u_{l}} \geq 0, \quad u=s \frac{u_{v}-u_{l}}{s_{v}-s_{l}}+\frac{s_{v} u_{l}-s_{l} u_{v}}{s_{v}-s_{l}} \leq 0 .
$$

The estimates follow from

$\left[u_{v} M_{v} / M+u_{l} M_{l} / M\right] \cdot\left[s_{v}-s_{l}\right]+\left[M_{v} / M+M_{l} / M\right] \cdot\left[u_{v} s_{l}-u_{l} s_{v}\right] \geq 0 \quad$ (where the equality sign is valid for $T=0)$ and

$\left[s_{v} M_{v} / M+s_{l} M_{l} / M\right] \cdot\left[u_{v}-u_{l}\right]+\left[M_{v} / M+M_{l} / M\right] \cdot\left[s_{v} u_{l}-s_{l} u_{v}\right] \leq 0 \quad$ (where the equality sign is valid for $T=T_{c}$ ).

The two obviously equivalent Equations (1.30) present the opportunity for proving the correctness of the reference data mentioned in Equations (1.8), (1.13), (1.17), (1.21) and (1.27). For example, it follows from $s\left(v_{c}, 0\right)=0$ that $u\left(v_{c}, 0\right)=\mu(0)=u_{l}(0)=-\left(u_{v}-u_{l}\right)(0) \quad$ with $\quad u_{v}(0)=0 \quad$ and from $s\left(v_{c}, T_{c}\right)=-\mathrm{d} \mu / \mathrm{d} T_{\mid c}+v_{c} \mathrm{~d} p / \mathrm{d} T_{\mid c}$ that $u\left(v_{c}, T_{c}\right)=0$. The data mentioned are thus reciprocal to one another. These data are thermodynamic reference values.

\subsection{Heat Capacity Relations}

The measurable heat capacity is defined by

$$
c(v, T)=\frac{\mathrm{d} u(v, T)}{\mathrm{d} T}=\frac{\mathrm{d} s(v, T)}{\mathrm{d} T} T=s(v, T)+\frac{\mathrm{d} s(v, T)}{\mathrm{d} T} T+\frac{\mathrm{d} f(v, T)}{\mathrm{d} T} .
$$

Calculation of the specific heat capacities requires the temperature derivatives of the quantities given in Equations (1.23) and (1.25). Taking into consideration Equations (3.1)-(3.3) below, the heat capacity relations are 


$$
\begin{aligned}
& c(T, v)=\frac{\mathrm{d}}{\mathrm{d} T}\left[\left[v_{v}+v_{l}-v-\frac{v_{v}-v_{l}}{\ln \left(v_{v} / v_{l}\right)}\right] \frac{\mathrm{d}(p / T)}{\mathrm{d}(1 / T)}\right]=-\frac{\mathrm{d}^{2} \mu}{\mathrm{d} T^{2}} T+v \frac{\mathrm{d}^{2} p}{\mathrm{~d} T^{2}} T \geq 0, \\
& c_{v, l}(T)=c(T, v)+\left(v_{v, l}-v\right)(\partial c(T, v) / \partial v)_{T}=-\frac{\mathrm{d}^{2} \mu}{\mathrm{d} T^{2}} T+v_{v, l} \frac{\mathrm{d}^{2} p}{\mathrm{~d} T^{2}} T \geq 0 .
\end{aligned}
$$

As the values of $c(T, v), s(T, v)$, and $\mathrm{d}^{2} p / \mathrm{d} T^{2}$ vanish at absolute zero, those of $(-\mathrm{d} \mu / \mathrm{d} T)$ and $\left(-\mathrm{d}^{2} \mu / \mathrm{d} T^{2}\right)$ also vanish there according to Equations (1.18) and (1.19). And as the value $\mathrm{d}^{2} \mu / \mathrm{d} T^{2}$ diverges at the critical point, the fluid heat capacity and the specific heat capacities also diverge there: $c\left(T_{c}, v\right)=c_{l}\left(T_{c}\right)=c_{v}\left(T_{c}\right) \rightarrow+\infty$.

Further expressions for the heat capacity and temperature derivatives of internal energies can be given as follows:

$$
\begin{aligned}
\frac{\mathrm{d} u(v, T)}{\mathrm{d} T}= & c(v, T)=c_{v} \frac{v-v_{l}}{v_{v}-v_{l}}+c_{l} \frac{v_{v}-v}{v_{v}-v_{l}} \\
= & {\left[\frac{\mathrm{d} u_{v}}{\mathrm{~d} T}+\frac{\mathrm{d} v_{v}}{\mathrm{~d} T} \frac{\mathrm{d}(p / T)}{\mathrm{d}(1 / T)}\right] \frac{v-v_{l}}{v_{v}-v_{l}}+\left[\frac{\mathrm{d} u_{l}}{\mathrm{~d} T}+\frac{\mathrm{d} v_{l}}{\mathrm{~d} T} \frac{\mathrm{d}(p / T)}{\mathrm{d}(1 / T)}\right] \frac{v_{v}-v}{v_{v}-v_{l}}, } \\
\frac{\mathrm{d} u_{v, l}}{\mathrm{~d} T}= & -T \frac{\mathrm{d}^{2} \mu}{\mathrm{d} T^{2}}+v_{v, l} \frac{\mathrm{d}^{2} p}{\mathrm{~d} T^{2}}-\frac{\mathrm{d} v_{v, l}}{\mathrm{~d} T} \frac{\mathrm{d}(p / T)}{\mathrm{d}(1 / T)} \\
= & c(v, T)+\left(v_{v, l}-v\right) \frac{c_{v}-c_{l}}{v_{v}-v_{l}}+\frac{\mathrm{d} v_{v, l}}{\mathrm{~d} T} \frac{u_{v}-u_{l}}{v_{v}-v_{l}} \\
= & c_{v} \frac{v-v_{l}}{v_{v}-v_{l}}+c_{l} \frac{v_{v}-v}{v_{v}-v_{l}}+\left(v_{v, l}-v\right) \frac{c_{v}-c_{l}}{v_{v}-v_{l}}+\frac{\mathrm{d} v_{v, l}}{\mathrm{~d} T} \frac{u_{v}-u_{l}}{v_{v}-v_{l}} . \\
= & c_{v, l}+\frac{\mathrm{d} v_{v, l}}{\mathrm{~d} T} \frac{u_{v}-u_{l}}{v_{v}-v_{l}}=c_{v, l}-\frac{\mathrm{d} v_{v, l}}{\mathrm{~d} T} \frac{\mathrm{d}(p / T)}{\mathrm{d}(1 / T)}, \\
& \frac{\mathrm{d}\left(u_{v}-u_{l}\right)}{\mathrm{d} T}=\left(c_{v}-c_{l}\right)+\frac{\mathrm{d}\left(v_{v}-v_{l}\right)}{\mathrm{d} T} \frac{u_{v}-u_{l}}{v_{v}-v_{l}} \\
= & \left(v_{v}-v_{l}\right) \frac{\mathrm{d}^{2} p}{\mathrm{~d} T^{2}} T-\frac{\mathrm{d}\left(v_{v}-v_{l}\right)}{\mathrm{d} T} \frac{\mathrm{d}(p / T)}{\mathrm{d}(1 / T)}, \\
\frac{\mathrm{d}\left(u_{v}+\right.}{\mathrm{d} T} & \left.u_{l}\right) \\
= & -2 \frac{\mathrm{d}^{2} \mu}{\mathrm{d} T^{2}} T+\left(v_{v}+v_{l}\right) \frac{\mathrm{d}^{2} p}{\mathrm{~d} T^{2}} T-\frac{\mathrm{d}\left(v_{v}+v_{l}\right)}{\mathrm{d} T} \frac{\mathrm{d}(p / T)}{\mathrm{d}(1 / T)}>0 .
\end{aligned}
$$

It holds that

$$
\begin{aligned}
-\left(u_{v}-u_{l}\right)(0) & =u_{l}(0)=u(0)=\left(u_{v}+u_{l}\right)(0)<u_{l}(T)<u(T)<\left(u_{v}+u_{l}\right)(T)<u\left(T_{c}\right) \\
& =\left(u_{v}+u_{l}\right)\left(T_{c}\right)=0
\end{aligned}
$$

and for $0<T<T_{c}$ that

$$
\left(u_{v}+u_{l}\right)-u=\left[\frac{v_{v}-v_{l}}{\ln \left(v_{v} / v_{l}\right)}-v_{c}\right]\left[-\frac{\mathrm{d}(p / T)}{\mathrm{d}(1 / T)}\right]>0 .
$$

In order to show that $\left(u_{v}+u_{l}\right)$ is a convexly curved temperature function, one has to prove that $\mathrm{d}^{2}\left(u_{v}+u_{l}\right) / \mathrm{d} T^{2}>0$ or that the straight line $u(0)\left(1-T / T_{c}\right)$ is above $\left(u_{v}+u_{l}\right)$. The condition of convexity for $\left(u_{v}+u_{l}\right)$ then reads $\left(u_{v}+u_{l}\right) \leq u(0)\left(1-T / T_{c}\right)$, which can be transformed to

$$
0 \leq u_{v}(T)+u_{l}(T)-u_{l}(0) \leq-u_{l}(0) \cdot T / T_{c} .
$$


Indeed, for $T>0$ the terms in Equation (1.37) are positive since $u_{v}(T)>0$ and $u_{l}(T)>u_{l}(0)$, which meets the condition mentioned. Likewise, the function $\left(u_{v}-u_{l}\right)(T)$ is concavely curved when its values are above the straight line $-u(0)\left(1-T / T_{c}\right)$, which means that $\left(u_{v}-u_{l}\right)(T) \geq-u(0)\left(1-T / T_{c}\right)$. Division by $-u(0)=\left(u_{v}-u_{l}\right)(0)>0$ gives the correct relations $1 \geq\left(u_{v}-u_{l}\right)(T) /\left(u_{v}-u_{l}\right)(0) \geq\left(1-T / T_{c}\right) \geq 0$ and thus confirms that the condition for concavity of $\left(u_{v}-u_{l}\right)$ is met.

From $c_{v, l}=\mathrm{d} u_{v, l} / \mathrm{d} T-\left(u_{v}-u_{l}\right) /\left(v_{v}-v_{l}\right) \cdot \mathrm{d} v_{v, l} / \mathrm{d} T$ and $\left(u_{v}-u_{l}\right) /\left(v_{v}-v_{l}\right)=-\mathrm{d}(p / T) / \mathrm{d}(1 / T)$ it follows that

$$
\begin{aligned}
\frac{c_{v}-c_{l}}{u_{v}-u_{l}} & =\frac{\mathrm{d} \ln \left[\left(u_{v}-u_{l}\right) /\left(v_{v}-v_{l}\right)\right]}{\mathrm{d} T} \\
& =\frac{\mathrm{d} \ln [-\mathrm{d}(p / T) / \mathrm{d}(1 / T)]}{\mathrm{d} T}=-\frac{\mathrm{d}(1 / T)}{\mathrm{d}(p / T)} \frac{\mathrm{d}^{2} p}{\mathrm{~d} T^{2}} T .
\end{aligned}
$$

It is of interest to take $T=0$ in entropy and heat capacity relations. This is immediately possible in the case of the entropy because Nernst's theorem states that the entropy vanishes at absolute zero, where only the condensed phase exists, and increases with the temperature:

$$
s(0, v)=s_{l}(0)=0, \quad(\partial s(T, v) / \partial T)_{v}=c(T, v) / T>0 .
$$

For $0 \leq T \leq T_{c}$ one thus gets

$$
0=s(0, v)=s_{l}(0)<s_{l}(T)<s(T, v)<s\left(T_{c}, v\right)=s_{l}\left(T_{c}\right)=s_{v}\left(T_{c}\right)<s_{v}(T) .
$$

Similarly, in the case of the heat capacity one gets for $0 \leq T<T_{c}$

$$
0=c(0, v)=c_{l}(0)<c_{l}(T)<c(T, v)<c_{v}(T) .
$$

The temperature derivatives of the chemical potential function $(-\mathrm{d} \mu / \mathrm{d} T)$ and the phase-specific entropies $s_{v, l}$ can be determined from measurements of $c(T, v), v_{v, l}(T)$ and $p(T)$ since

$$
\begin{gathered}
-\frac{\mathrm{d}^{2} \mu}{\mathrm{d} T^{2}}=\frac{c(T, v)}{T}-v \frac{\mathrm{d}^{2} p}{\mathrm{~d} T^{2}}>0, \\
\frac{\mathrm{d} s_{v, l}}{\mathrm{~d} T}=\frac{c(T, v)}{T}+\left(v_{v, l}-v\right) \frac{\mathrm{d}^{2} p}{\mathrm{~d} T^{2}}+\frac{\mathrm{d} v_{v, l}}{\mathrm{~d} T} \frac{\mathrm{d} p}{\mathrm{~d} T} .
\end{gathered}
$$

With the result $(-\mathrm{d} \mu / \mathrm{d} T)>0$ one obtains from Equations (1.23) the relations

$$
s_{l}-v_{l} \frac{\mathrm{d} p}{\mathrm{~d} T}=s_{v}-v_{v} \frac{\mathrm{d} p}{\mathrm{~d} T}=s-v \frac{\mathrm{d} p}{\mathrm{~d} T}=-\frac{\mathrm{d} \mu}{\mathrm{d} T}>0 .
$$

These state that the entropy values are always positive and greater than the product of the volumes and vapor pressure coefficient. From relations (1.43), in turn, one can derive the following relations:

$$
\frac{s_{l}}{v_{l}}>\frac{s}{v}>\frac{s_{v}}{v_{v}}>\frac{\mathrm{d} p}{\mathrm{~d} T}>0 .
$$

Temperature properties of the phase-specific heats are similarly derived. With the result $\left(-\mathrm{d}^{2} \mu / \mathrm{d} T^{2}\right)>0$ one obtains from Equations (1.32) the relations 


$$
c_{l}-v_{l} \frac{\mathrm{d}^{2} p}{\mathrm{~d} T^{2}} T=c-v \frac{\mathrm{d}^{2} p}{\mathrm{~d} T^{2}} T=c_{v}-v_{v} \frac{\mathrm{d}^{2} p}{\mathrm{~d} T^{2}} T=-\frac{\mathrm{d}^{2} \mu}{\mathrm{d} T^{2}} T>0,
$$

from which in turn the relations

$$
\frac{c_{l}}{v_{l}}>\frac{c}{v}>\frac{c_{v}}{v_{v}}>\frac{\mathrm{d}^{2} p}{\mathrm{~d} T^{2}}>0
$$

can be derived. Experimental verification of $C_{V} / V=c / v>\mathrm{d}^{2} p / \mathrm{d} T^{2} \cdot T$ also proves confirmation of $\left(-\mathrm{d}^{2} \mu / \mathrm{d} T^{2}\right)>0$.

\subsection{Chemical Potential Relations}

The identities

$$
\begin{gathered}
\frac{x_{v} y_{l}-x_{l} y_{v}}{y_{v}-y_{l}}=-x_{v, l}+y_{v, l}\left(\frac{\partial x}{\partial y}\right)_{T}, \\
\frac{\mathrm{d}}{\mathrm{d} T} \frac{x_{v} y_{l}-x_{l} y_{v}}{y_{v}-y_{l}}=-\frac{\mathrm{d} x_{v, l}}{\mathrm{~d} T}+\frac{\mathrm{d} y_{v, l}}{\mathrm{~d} T}\left(\frac{\partial x}{\partial y}\right)_{T}+y_{v, l} \frac{\mathrm{d}}{\mathrm{d} T}\left(\frac{\partial x}{\partial y}\right)_{T}
\end{gathered}
$$

are now used to put the chemical potential functions in explicit form as energy functions:

$$
\begin{gathered}
\mu=f_{v, l}+v_{v, l} p=u_{v, l}-s_{v, l} T+v_{v, l} p<0, \\
\frac{\mathrm{d} \mu}{\mathrm{d} T}=-s_{v, l}+v_{v, l} \frac{\mathrm{d} p}{\mathrm{~d} T}=\frac{\mathrm{d} f_{v, l}}{\mathrm{~d} T}+\frac{\mathrm{d} v_{v, l}}{\mathrm{~d} T} p+v_{v, l} \frac{\mathrm{d} p}{\mathrm{~d} T}<0, \\
\frac{\mathrm{d}(\mu / T)}{\mathrm{d}(1 / T)}=u_{v, l}+v_{v, l} \frac{\mathrm{d}(p / T)}{\mathrm{d}(1 / T)}=\frac{\mathrm{d}\left(f_{v, l} / T\right)}{\mathrm{d}(1 / T)}-\frac{\mathrm{d} v_{v, l} l}{\mathrm{~d} T} T p+v_{v, l} \frac{\mathrm{d}(p / T)}{\mathrm{d}(1 / T)}<0, \\
\frac{\mathrm{d}^{2} \mu}{\mathrm{d} T^{2}}=-\frac{c_{v, l}}{T}+v_{v, l} \frac{\mathrm{d}^{2} p}{\mathrm{~d} T^{2}}=-\frac{\mathrm{d} s_{v, l}}{\mathrm{~d} T}+\frac{\mathrm{d} v_{v, l}}{\mathrm{~d} T} \frac{\mathrm{d} p}{\mathrm{~d} T}+v_{v, l} \frac{\mathrm{d}^{2} p}{\mathrm{~d} T^{2}}<0 .
\end{gathered}
$$

The relations state that $\mu$ is a negative, concavely curved function, decreasing with increasing $T$. For $\mu$ as a function of measurable quantities see Equations (3.15) and (3.16) below.

\section{Internal Fluid Energy as a Function of the Condensation Energy}

It can be shown that the fluid energy $u(T, v)$ can be expressed in terms of the condensation energy $\left[-\left(u_{v}-u_{l}\right)\right]$ :

$$
\begin{aligned}
u & =\frac{\mathrm{d}(\mu / T)}{\mathrm{d}(1 / T)}-v \frac{\mathrm{d}(p / T)}{\mathrm{d}(1 / T)}=\frac{\mathrm{d}(p / T)}{\mathrm{d}(1 / T)}\left[\frac{\mathrm{d}(\mu / T)}{\mathrm{d}(p / T)}-v\right] \\
& =\frac{-\left(u_{v}-u_{l}\right)}{v_{v}-v_{l}}\left[\frac{u_{v} v_{l}-u_{l} v_{v}}{u_{v}-u_{l}}-v\right] \\
& =-\left(u_{v}-u_{l}\right)\left[\frac{v_{l}-v}{v_{v}-v_{l}}-\frac{u_{l}}{u_{v}-u_{l}}\right] \\
& =-\left(u_{v}-u_{l}\right)\left[\frac{v_{v}-v}{v_{v}-v_{l}}-\frac{u_{v}}{u_{v}-u_{l}}\right] .
\end{aligned}
$$

Since the temperature coefficients $\left(v-v_{l}\right) /\left(v_{v}-v_{l}\right)$ and $\left(v_{v}-v\right) /\left(v_{v}-v_{l}\right)$ 
are represented by $M_{v} / M$ and $M_{l} / M$, respectively, the mass distributions $M_{v} / M=0$ and $M_{l} / M=1$ at $T=0$ give the three relations $u(0)=u_{l}(0)$, $u(0)=-\left(u_{v}-u_{l}\right)(0)+u_{v}(0), u(0)=-\left(u_{v}-u_{l}\right)(0)$, and the distributions $M_{v} / M=M_{l} / M=1 / 2$ at $T=T_{c}$ give the three relations $u\left(T_{c}\right)=\left(u_{v}-u_{l}\right)\left(T_{c}\right) / 2+u_{l}\left(T_{c}\right), u\left(T_{c}\right)=-\left(u_{v}-u_{l}\right)\left(T_{c}\right) / 2+u_{v}\left(T_{c}\right)$, $u\left(T_{c}\right)=u_{v}\left(T_{c}\right) / 2+u_{l}\left(T_{c}\right) / 2$. One thus obtains the following characteristic thermodynamic reference values, which are valid for every gas:

$$
u(0)=u_{l}(0)=-\left(u_{v}-u_{l}\right)(0), u_{v}(0)=0, u\left(T_{c}\right)=u_{l}\left(T_{c}\right)=u_{v}\left(T_{c}\right)=0 .
$$

\section{Internal Fluid Energy as an Expression of Measurable Quantities}

Endeavors to publish data of the functions $u\left(v_{c}, T\right)$ and $u_{v, l}(T)$ are prominent in the current literature. The energy equations can be written in the form

$$
u\left(v_{c}, T\right)=\left[\frac{\mathrm{d}(\mu / T)}{\mathrm{d}(p / T)}-v_{c}\right] \frac{\mathrm{d}(p / T)}{\mathrm{d}(1 / T)} \leq 0, u_{v, l}(T)=\left[\frac{\mathrm{d}(\mu / T)}{\mathrm{d}(p / T)}-v_{v, l}\right] \frac{\mathrm{d}(p / T)}{\mathrm{d}(1 / T)} .
$$

According to Equations (1.20)-(1.22) the diffential quotient $\mathrm{d}(\mu / T) / \mathrm{d}(p / T)$ is a positive volume quantity monotonically decreasing with increasing $T$ from high values $v_{v}(T)$ near absolute zero to the lowest value $v_{c}$ at the critical point. The expression of measurable volumes $v_{v}(T)$ and $v_{l}(T)$ instead of $\mathrm{d}(\mu / T) / \mathrm{d}(p / T)$ as published in (2015) [3] reads:

$$
\frac{\mathrm{d}(\mu / T)}{\mathrm{d}(p / T)}=v_{v}+v_{l}-\frac{v_{v}-v_{l}}{\ln \left(v_{v} / v_{l}\right)} \text {. }
$$

It is symmetric in the variables and linear in both $v_{V}$ and $v_{b}$ and at the critical point it yields $V_{c}$. Then inserting the solution (3.2) in the internal energy Equations (3.1) yields the results

$$
\begin{aligned}
& u\left(T, v_{c}\right)=\left[\frac{v_{v}-v_{l}}{\ln \left(v_{v} / v_{l}\right)}-v_{v}-v_{v}+v_{c}\right] \cdot\left[-\frac{\mathrm{d}(p / T)}{\mathrm{d}(1 / T)}\right] \leq 0, \\
& u_{v, l}(T)=\left[\frac{v_{v}-v_{l}}{\ln \left(v_{v} / v_{l}\right)}-v_{l, v}\right] \cdot\left[-\frac{\mathrm{d}(p / T)}{\mathrm{d}(1 / T)}\right] .
\end{aligned}
$$

These equations state that the internal energies can be expressed in terms of the measurable quantities, phase-specific volumes and vapor pressure, and are given by absolute figures; in particular, it holds that $u_{v}\left(T_{c}\right)=u_{l}\left(T_{c}\right)=0$ since $\left(v_{v}-v_{l}\right) / \ln \left(v_{v} / v_{l}\right)=v_{c}$ and $v_{v}=v_{l}=v_{c}$ at $T=T_{c}$.

If the functions $u_{v}$ and $u_{l}$ are expressed as dependent on the volume ratio $z=v_{v} / v_{l}$, one obtains for the energy ratios $u_{v} /\left(u_{v}-u_{l}\right)$ and $-u_{l} /\left(u_{v}-u_{l}\right)$ according to Equations (1.28) the following relations:

$$
\begin{aligned}
& 0 \leq \frac{u_{v}}{u_{v}-u_{l}}=\frac{1}{\ln z}-\frac{1}{z-1} \leq \frac{1}{2} \text { for } 0 \leq T \leq T_{c} \text { and } z=\frac{v_{v}(T)}{v_{l}(T)} \geq 1, \\
& \frac{1}{2} \leq \frac{-u_{l}}{u_{v}-u_{l}}=-\frac{1}{\ln z}+\frac{z}{z-1} \leq 1 .
\end{aligned}
$$


The sum of them is, of course, equal to 1. Equations (3.4) allow one to calculate the relation between the energy ratio $\eta=u_{v} / u_{l}$ and volume ratio $z$ of fluid particles in the vapor and liquid spaces, viz.

$$
0 \geq \eta=\frac{u_{v}}{u_{l}}=\frac{z-1-\ln z}{z-1-z \ln z} \geq-1 \text { for } 0 \leq T \leq T_{c} \text { and } z=\frac{v_{v}(T)}{v_{l}(T)} \geq 1 .
$$

According to Equations (1.17) and (1.8), the energy ratio $\eta$ assumes the value 0 at absolute zero (where $z \rightarrow \infty$ ) and, respectively, the value -1 at the critical point (where $z=1$ ). The relation $\eta(z)$ represents a universal law of the two-phase equilibrium of real gases.

Calculation of the energy ratio $\left(u_{v}+u_{l}\right) /\left(u_{v}-u_{l}\right)$ as a function of $z$ starts from Equation (1.20) or from $(\eta+1) /(\eta-1)$ and ends in any case with the result

$$
-1 \leq \frac{u_{v}+u_{l}}{u_{v}-u_{l}}=\frac{2}{\ln z}-\frac{z+1}{z-1} \leq 0 \text { for } 0 \leq T \leq T_{c} \text { and } z=\frac{v_{v}}{v_{l}} \geq 1 .
$$

Rigorous thermodynamic calculations combine Equations (1.8) and (1.44) and yield the following relations for the internal energy and entropy:

$$
-1 \leq u_{v} / u_{l}<0,1 \leq s_{v} / s_{l} \leq v_{v} / v_{l} \text { for } T \leq T_{c} .
$$

The constraints (3.7) state that the ratios of absolute energy and entropy for vapor and liquid are restricted within certain limits for temperatures in the two-phase region. And combining Equations (1.20), (1.22) and (3.2) gives

$$
\begin{aligned}
& v_{v}+v_{l}-\frac{u_{v} v_{v}-u_{l} v_{l}}{u_{v}-u_{l}}=\frac{u_{v} v_{l}-u_{l} v_{v}}{u_{v}-u_{l}}=v_{v}+v_{l}-\frac{v_{v}-v_{l}}{\ln \left(v_{v} / v_{l}\right)}, \\
& \frac{u_{v} v_{v}-u_{l} v_{l}}{u_{v}-u_{l}}=\frac{v_{v}-v_{l}}{\ln \left(v_{v} / v_{l}\right)} .
\end{aligned}
$$

Algebraic rearrangement of Equation (3.8) leads to a data criterion for consistent thermodynamic values $v_{v}(T), v_{l}(T), u_{v}(T)$ and $u_{l}(T)$, which is valid for $T \leq T_{c}$ and reads:

$$
\left[\frac{u_{v}}{u_{v}-u_{l}}+\frac{v_{l}}{v_{v}-v_{l}}\right] \cdot \ln \left(\frac{v_{v}}{v_{l}}\right)=\left[\frac{u_{l}}{u_{v}-u_{l}}+\frac{v_{v}}{v_{v}-v_{l}}\right] \cdot \ln \left(\frac{v_{v}}{v_{l}}\right)=1 .
$$

In turn, from Equations (3.9) one obtains

$$
\begin{aligned}
& u_{v}=\frac{u_{v}-u_{l}}{\ln \left(v_{v} / v_{l}\right)}-v_{l} \frac{u_{v}-u_{l}}{v_{v}-v_{l}} \geq 0, u_{l}=\frac{u_{v}-u_{l}}{\ln \left(v_{v} / v_{l}\right)}-v_{v} \frac{u_{v}-u_{l}}{v_{v}-v_{l}} \leq 0, \\
& \left(u_{v}-u_{l}\right)=\left(h_{v}-h_{l}\right)-\left(v_{v}-v_{l}\right) p=\left(s_{v}-s_{l}\right) T-\left(v_{v}-v_{l}\right) p=-\left(v_{v}-v_{l}\right) \frac{\mathrm{d}(p / T)}{\mathrm{d}(1 / T)} \geq 0 .
\end{aligned}
$$

Equations (3.9) and (3.10) clearly state that, if table data $T, p, V_{v}, V_{p}\left(h_{v}-h_{l}\right)$, or $\left(s_{v}-s_{l}\right)$ and $\left(u_{v}-u_{l}\right)$ are thermodynamically consistent, then the internal energies, $u_{v}, u_{p} \quad u=u_{v}\left(v_{c}-v_{l}\right) /\left(v_{v}-v_{l}\right)+u_{l}\left(v_{v}-v_{c}\right) /\left(v_{v}-v_{l}\right)$, can be given absolutely. An entropy data criterion of the same kind as for the internal energy can also be formulated. To this end one has to confirm the validity of the relation. 


$$
\frac{s_{v, l}+\mu / T-\left[v_{v}+v_{l}-\left(v_{v}-v_{l}\right) / \ln \left(v_{v} / v_{l}\right)\right] p / T}{s_{v}-s_{l}}=\frac{u_{v, l}}{u_{v}-u_{l}}
$$

to obtain with $u_{v, l} /\left(u_{v}-u_{l}\right)=1 / \ln \left(v_{v} / v_{l}\right)-v_{l, v} /\left(v_{v}-v_{l}\right)$ the criterion desired:

$$
\left[\frac{s_{v, l}}{s_{v}-s_{l}}+\frac{v_{l, v}}{v_{v}-v_{l}}+\frac{\mu}{\left(s_{v}-s_{l}\right) T}-\left[\frac{v_{v}+v_{l}}{v_{v}-v_{l}}-\frac{1}{\ln \left(v_{v} / v_{l}\right)}\right] \frac{p / T}{\mathrm{~d} p / \mathrm{d} T}\right] \cdot \ln \left(\frac{v_{v}}{v_{l}}\right)=1 \text {. }
$$

This relation allows, in principle, to give the chemical potential in terms of $p$, $v_{v, l}$ and $s_{v, l}$ as follows:

$$
\mu=-S_{v, l} T-v_{l, v} \frac{\mathrm{d} p}{\mathrm{~d} T} T+\frac{v_{v}-v_{l}}{\ln \left(v_{v} / v_{l}\right)} \frac{\mathrm{d} p}{\mathrm{~d} T} T+\left[v_{v}+v_{l}-\frac{v_{v}-v_{l}}{\ln \left(v_{v} / v_{l}\right)}\right] p .
$$

The entropy expressions read

$$
0 \leq s\left(v_{c}, T\right)=-\frac{\mathrm{d} \mu}{\mathrm{d} T}+v_{c} \frac{\mathrm{d} p}{\mathrm{~d} T}=s_{l}+\left(v_{c}-v_{l}\right) \frac{\mathrm{d} p}{\mathrm{~d} T}=s_{v}-\left(v_{v}-v_{c}\right) \frac{\mathrm{d} p}{\mathrm{~d} T} .
$$

It holds that $-S_{v, l} T-v_{l, v} \frac{\mathrm{d} p}{\mathrm{~d} T} T=-s T-\left(v_{v}+v_{l}-v_{c}\right) \frac{\mathrm{d} p}{\mathrm{~d} T} T$ and

$$
\mu=-s T-\left[v_{v}+v_{l}-\frac{v_{v}-v_{l}}{\ln \left(v_{v} / v_{l}\right)}\right] \frac{\mathrm{d} p}{\mathrm{~d} T} T+v_{c} \frac{\mathrm{d} p}{\mathrm{~d} T} T+\left[v_{v}+v_{l}-\frac{v_{v}-v_{l}}{\ln \left(v_{v} / v_{l}\right)}\right] p .
$$

According to relations (1.39) one has $s T=T \int_{0}^{T} \frac{\mathrm{d} s}{\mathrm{~d} T} \mathrm{~d} T=T \int_{0}^{T} \frac{c}{T} \mathrm{~d} T$. The chemical potential can thus be determined, on the one hand, by measuring the two-phase heat capacity, phase-specific volumes and vapor pressure:

$$
\mu(T)=-T \int_{0}^{T} \frac{c\left(T, v_{c}\right)}{T} \mathrm{~d} T+v_{c} \frac{\mathrm{d} p}{\mathrm{~d} T} T-\left[v_{v}+v_{l}-\frac{v_{v}-v_{l}}{\ln \left(v_{v} / v_{l}\right)}\right]\left[-\frac{\mathrm{d}(p / T)}{\mathrm{d}(1 / T)}\right],
$$

or, on the other, by measuring the phase-specific volumes and vapor pressure only:

$$
\mu(T)=T\left[\frac{\mu\left(T_{c}\right)}{T_{c}}-\int_{p / T}^{p\left(T_{c}\right) / T_{c}}\left[v_{v}+v_{l}-\frac{v_{v}-v_{l}}{\ln \left(v_{v} / v_{l}\right)}\right] \mathrm{d} \frac{p}{T}\right]<0, \frac{\mu_{c}}{T_{c}}=-S_{c}+v_{c} \frac{p_{c}}{T_{c}} .
$$

The energy sum $(\mu+s T)$ is also measurable and calculable and it holds that

$$
\mu+s T=\left[\frac{v_{v}-v_{l}}{\ln \left(v_{v} / v_{l}\right)}-v_{v}-v_{l}\right] \cdot\left[-\frac{\mathrm{d}(p / T)}{\mathrm{d}(1 / T)}\right]+v_{c} \frac{\mathrm{d} p}{\mathrm{~d} T} T=u+v_{c} p .
$$

From $\mathrm{d}(\mu+s T) / \mathrm{d} T=c+v_{c} \mathrm{~d} p / \mathrm{d} T>0$ and $\mathrm{d}^{2}(\mu+s T) / \mathrm{d} T^{2}=\mathrm{d} c / \mathrm{d} T+v_{c} \mathrm{~d}^{2} p / \mathrm{d} T^{2}>0$ it is concluded that the energy sum $\mu+s T=u+v_{c} p$ is a convex temperature function strongly increasing from $\mu(0)=-\left(u_{v}-u_{l}\right)(0)<0$ at absolute zero to $\mu_{c}+s_{c} T_{c}=v_{c} p_{c}>0$ at the critical point.

\section{Phase-Specific Energy as a Function of the Evaporation Energy}

The Carnot-Clapeyron-Clausius equation 


$$
u_{v}-u_{l}=\left(v_{v}-v_{l}\right)[-\mathrm{d}(p / T) / \mathrm{d}(1 / T)]
$$

suggests a unique relation between the energy density and particle density in the coexisting bulk phases, vapor and liquid. As the local interaction potentials in the partial volumens $V_{V}$ and $V_{l}$ are unique functions of the local particle density and determine the values $u_{V}$ and $u_{b}$ respectively, it should be possible by means of a density coefficient and a temperature function to find suitable ansatzes for the functions $u_{v, l}$ depending on the density coefficient and temperature function. As density coefficient, a function $\rho$ of the density variable $z=v_{v} / v_{l}$ is now chosen, and as temperature function the evaporation energy $\left(u_{v}-u_{l}\right)$. The ansatz proposed for the energy in the vapor phase is then $u_{v}=\rho(1 / z) \cdot\left(u_{v}-u_{l}\right)$. The energy in the condensed phase must be $u_{l}=-\rho(z) \cdot\left(u_{v}-u_{l}\right)$ since the transfer of a particle from $V_{V}$ to $V_{l}$ causes the value $u_{V}$ to change to $u_{p}$, the density variable from $1 / z$ to $z$ and the phase transition energy from $\left(u_{v}-u_{l}\right)$ to $\left(u_{l}-u_{v}\right)=-\left(u_{v}-u_{l}\right)$, while the form of $\rho$ is preserved because the functional density dependence of the microscopic interaction forces is of course phase-invariant. The conjectures [2]

$$
u_{v}=\rho(1 / z) \cdot\left(u_{v}-u_{l}\right), \quad u_{l}=-\rho(z) \cdot\left(u_{v}-u_{l}\right)
$$

then yield, on the one hand, the reference values $0=u_{v}\left(T_{c}\right)=u_{l}\left(T_{c}\right)=\frac{1}{2}\left[u_{v}\left(T_{c}\right)+u_{l}\left(T_{c}\right)\right]=u\left(v_{c}, T_{c}\right)$ and, on the other, for the density coefficient the equation

$$
\rho(1 / z)+\rho(z)=1
$$

and because $u_{v}(0)=0$ and $u_{v}\left(T_{c}\right) / u_{l}\left(T_{c}\right)=-1$ the boundary conditions

$$
0 \leq \rho(1 / z) / \rho(z) \leq 1 \text {. }
$$

The functional Equation (4.3) for $\rho$ under condition (4.4) is satisfied by

$$
0 \leq \rho(1 / z)=\frac{1}{\ln z}-\frac{1}{z-1} \leq \frac{1}{2} \leq \rho(z)=-\frac{1}{\ln z}+\frac{z}{z-1} \leq 1 .
$$

From Equation (4.2) it follows that

$$
0 \leq \frac{u_{v}}{u_{v}-u_{l}}=\frac{1}{\ln z}-\frac{1}{z-1} \leq \frac{1}{2} \leq-\frac{1}{\ln z}+\frac{z}{z-1}=\frac{-u_{l}}{u_{v}-u_{l}} \leq 1 .
$$

The relations (4.6) and (3.4) are identical and valid for $0 \leq T \leq T_{c}$ and $z=v_{v}(T) / v_{l}(T) \geq 1$

\section{Results and Discussion}

The paper treats thermodynamic properties of the saturated fluid. It is shown that the fluid state is completely determined by the internal energy and entropy in the vapor and liquid spaces. The ratios of the absolute phase-specific internal energies and entropies are restricted within certain limits. If for temperatures $T \subset\left[T_{1}, T_{2}\right]$ measured saturation data of the vapor pressure, reciprocal phase-specific densities and isothermal transient energy obey the data criterion Equation (3.9), then the internal energy as a function of $T$ calculated according 
to Equations (3.10) is an absolute thermodynamic quantity. This fundamental procedure in gaining thermodynamic data excludes any application of so-called fiducial reference data since they cannot yield correct values. As the state of saturation is maintained, it is not possible to distinguish between a constant pressure and a constant volume condition. This is the characteristic difference between the heat capacities of a two-phase and a one-phase fluid. There is only one phase-specific heat capacity in the vapor space, $c_{v}(T)$, and one in the liquid space, $c_{l}(T)$. The measurable heat capacity of the fluid, $C\left(v_{c}, T\right)=M c\left(v_{c}, T\right)=M_{v} c_{v}+M_{l} c_{l}$, can also be calculated. Further results are the concavity of the positive, measurable temperature function $\left(u_{v}-u_{l}\right)$ and the convexity of the negative temperature functions $\left(u_{v}+u_{l}\right)$. The chemical potential is a negative, measurable and calculable temperature function. The ratios of phase-specific energies to isothermal transient

energies obey the relations $0 \leq u_{v} /\left(u_{v}-u_{l}\right) \leq \frac{1}{2} \leq u_{l}\left(u_{l}-u_{v}\right) \leq 1$, which should be heeded when a state chart of the fluid under consideration is constructed.

\section{Acknowledgements}

The author is grateful to A. M. Nicol for the English translation.

\section{References}

[1] Gibbs, J.W. (1873) A Method of Geometrical Representation of the Thermodynamic Properties of Substances by Means of Surfaces. Transactions of the Connecticut Academy, 2, 382-404. http://www3.nd.edu/-powers/ame.20231/gibbs1873b.pdf

[2] Elsner, A. (1988) Thermodynamic Representation of the State of a Saturated Fluid. Physics Letters A, 130, 225-233. https://doi.org/10.1016/0375-9601(88)90598-1

[3] Elsner, A. (2015) Thermodynamic Equilibrium of the Saturated Fluid with a Free Surface Area and the Internal Energy as a Function of the Phase-Specific Volumes and Vapor Pressure. Engineering, 7, 577-596.

https://doi.org/10.4236/eng.2015.79053

[4] Elsner, A. (2012) Applied Thermodynamics of the Real Gas with Respect to the Thermodynamic Zeros of the Entropy and Internal Energy. Physica B: Physics of Condensed Matter, 407, 1055-1067. https://doi.org/10.1016/j.physb.2011.12.118

[5] Strunk, Ch. (2015) Moderne Thermodynamik: Von einfachen Systemen zu Nanostrukturen. 32. https://doi.org/10.1515/9783110371062

[6] Lauth, J.G. and Kowalczyk, J. (2015) Thermodynamik. Springer-Verlag, Berlin Heidelberg, 67. https://doi.org/10.1007/978-3-662-46229-4

[7] Callen, H.B. (1960) Thermodynamics. John Wiley \& Sons, Hoboken, 52.

[8] Johnson, V.J. (1961) Properties of Materials at Low Temperature (Phase 1). National Bureau of Standards, Cryogenic Engineering Laboratory, Pergamon Press, Oxford, London, New York, Paris.

[9] Journal of Physical and Chemical Reference Data, Annual Series of Properties of Gases, published since 1972.

[10] IUPAC Commission on Thermodynamics (1972) International Thermodynamic Tables of the Fluid State. Blackwell Scientific Publications, Hoboken, Vol. 1. 
[11] Selover, T.B. (1987) A Series of Property Tables. Hemisphere Publishing Corporation, New York.

[12] Reynolds, W.C. (1979) Thermodynamic Properties in SI. Graphs, Tables and Computational Equations for 40 Substances. The Department of Mechanical Engineering, Stanford University, Stanford.

[13] Grigull, U. and Schmidt, E. (1989) Properties of Water and Steam in SI-Units. Springer-Verlag, Berlin, Heidelberg, New York.

[14] Wagner, W. and Pruss, A. (1993) International Equations for the Saturation Properties of Ordinary Water Substance. Revised According to the International Temperature Scale of 1990. Addendum to J. Phys. Chem. Ref. Data 16, 893 (1987). Journal of Physical and Chemical Reference Data, 22, 783.

https://doi.org/10.1063/1.555926

[15] Kohlrausch, F. (1996) Praktische Physik. Band 3, B. G. Teubner Stuttgart.

[16] Feistel, R. and Wagner, W. (2006) A New Equation of State for $\mathrm{H}_{2} \mathrm{O}$ Ice Ih. Journal of Physical and Chemical Reference Data, 35, 1021-1047. https://doi.org/10.1063/1.2183324

[17] Elsner, A. (2014) The Dominant Role of the Chemical Potential for Driving Currents in Oceans and Air. Journal of Geoscience and Environment Protection, 2 $117-125$.

[18] Pethick, C.J. and Smith, H. (2002) Bose-Einstein Condensation in Dilute Gases. Cambridge University Press, Cambridge.

[19] Elsner, A. (2017) Absolute Internal Energy of the Real Gas. Engineering, 9, 361-375. 


\section{Appendix: Comments on the Internationally Accepted Equations for the Saturation Properties of Water}

The International Association for the Properties of Water and Steam (IAPWS) [13] [14] provides internationally accepted formulations for the properties of water. There are special correlation equations for the vapor-liquid saturation properties of water. Formulas are given for the vapor pressure $p$, phase-specific volumes $v_{v, l}$, internal energies $u_{v, l}$, and entropies $s_{v, l}$ as functions of the saturation temperature $T$. This affords a unique description of the temperature dependence of every property of the saturated water.

This study treats the IAPWS equations in the framework of thermodynamics. The IAPWS equations read, on the one hand [13] [14],

$$
u_{v, l}=\alpha-v_{v, l} \frac{\mathrm{d}(p / T)}{\mathrm{d}(1 / T)}, \quad s_{v, l}=\phi+v_{v, l} \frac{\mathrm{d} p}{\mathrm{~d} T},
$$

where the so-called auxiliary quantities $\alpha$ and $\phi$ are given as functions of the temperature $T$; and, on the other, in terms of the thermodynamic fundamental equation,

$$
u_{v, l}-s_{v, l} T=\mu-v_{v, l} p,
$$

where $\mu$ is the chemical potential of the saturated fluid. Equations (A1) and (A2) are related as follows:

$$
\alpha-\phi T=\mu, \quad \alpha=\frac{\mathrm{d}(\mu / T)}{\mathrm{d}(1 / T)}, \quad \phi=-\frac{\mathrm{d} \mu}{\mathrm{d} T} .
$$

Let us now investigate the thermodynamic conditions that have to be satisfied by the temperature functions $\alpha$ and $\phi$ if they are to define the two-phase chemical potential $\mu$ according to Equations (A3). First a few thermodynamic relations are taken and then transformed into IAPWS parlance. The Carnot-Clapeyron-Clausius equations read

$$
\frac{u_{v}-u_{l}}{v_{v}-v_{l}}=-\frac{\mathrm{d}(p / T)}{\mathrm{d}(1 / T)}, \quad \frac{s_{v}-s_{l}}{v_{v}-v_{l}}=\frac{\mathrm{d} p}{\mathrm{~d} T} .
$$

These are satisfied by Equations (A1). The Gibbs-Duhem equations read

$$
\frac{\mathrm{d} u_{v, l}}{\mathrm{~d} T}+\frac{\mathrm{d} v_{v, l}}{\mathrm{~d} T} p=\frac{\mathrm{d} s_{v, l}}{\mathrm{~d} T} T .
$$

They are satisfied by Equations (A1) if the following relation between $\alpha$ and $\phi$ is valid:

$$
\frac{\mathrm{d} \alpha}{\mathrm{d} T}=\frac{\mathrm{d} \phi}{\mathrm{d} T} T .
$$

Note that Equation (A6) is implicitly contained in relations (A3) since $\mathrm{d} \mu / \mathrm{d} T=\mathrm{d} \alpha / \mathrm{d} T-\mathrm{d} \phi / \mathrm{d} T \cdot T-\phi=-\phi$ and $\mathrm{d}(\mu / T) / \mathrm{d}(1 / T)=\mu-\mathrm{d} \mu / \mathrm{d} T \cdot T=(\alpha-\phi T)+\phi T=\alpha$. Relations (A4) and (A5) therefore afford nothing new, but merely confirm relations (A3). The mathematical structures of Equations (A1) thus conform to the thermodynamic 
internal energy and entropy expressions in respect of Equations (A3).

Condition (A6) is numerically satisfied by the formulas in [13] [14] for $\alpha(T)$ and $\phi(T)$, whose temperature dependences are expressed as follows:

$$
\begin{gathered}
\alpha[\mathrm{J} / \mathrm{g}]=d_{\alpha}+d_{1} \theta^{-19}+d_{2} \theta+d_{3} \theta^{4.5}+d_{4} \theta^{5}+d_{5} \theta^{54.5}, \text { where } \theta \equiv \frac{T}{T_{c}}, \\
\phi[\mathrm{J} / \mathrm{g} \cdot \mathrm{K}]=\left[d_{\phi}+\frac{19}{20} d_{1} \theta^{-20}+d_{2} \ln \theta+\frac{9}{7} d_{3} \theta^{3.5}+\frac{5}{4} d_{4} \theta^{4}+\frac{109}{107} d_{5} \theta^{53.5}\right] \frac{1}{T_{c}},
\end{gathered}
$$

where $\left[d_{\alpha}, d_{\phi}\right]=[-1.135905627715 \mathrm{E} 3,2.3195246 \mathrm{E} 3], \quad T_{c}=647.096 \mathrm{~K}$,

$$
\begin{aligned}
{\left[d_{1}, d_{2}, d_{3}, d_{4}, d_{5}\right]=} & {[-5.65134998 \mathrm{E}-8,2.69066631 \mathrm{E} 3,1.27287297 \mathrm{E} 2,} \\
& -1.35003439 \mathrm{E} 2,9.81825814 \mathrm{E}-1] .
\end{aligned}
$$

The vapor pressure $p$ and chemical potential $\mu$ obey for temperatures $T \leq T_{c}$ the following relations:

$$
\begin{aligned}
& p>0, \quad \frac{\mathrm{d} p}{\mathrm{~d} T}>0, \quad \frac{\mathrm{d}(p / T)}{\mathrm{d}(1 / T)}<0, \quad \frac{\mathrm{d}^{2} p}{\mathrm{~d} T^{2}}>0, \\
& \mu<0, \quad \frac{\mathrm{d} \mu}{\mathrm{d} T}<0, \quad \frac{\mathrm{d}(\mu / T)}{\mathrm{d}(1 / T)}<0, \quad \frac{\mathrm{d}^{2} \mu}{\mathrm{d} T^{2}}<0 .
\end{aligned}
$$

Relations (A10) then lead to further conditions for $\alpha$ and $\phi$ :

$$
\alpha<0, \quad \frac{\mathrm{d} \alpha}{\mathrm{d} T}>0, \quad \phi>0, \quad \frac{\mathrm{d} \phi}{\mathrm{d} T}>0 .
$$

Accordingly, $\alpha$ must be a negative function increasing as $T$, and $\phi$ must be a positive function likewise increasing as $T$. It is found that $\mathrm{d} \alpha / \mathrm{d} T>0$, but not that $\alpha<0$ for all $T$ in the range $\left[T_{t}, T_{c}\right]$; similarly, it does hold that $\mathrm{d} \phi / \mathrm{d} T>0$, but not that $\phi>0$ for all $T \subset\left[T_{t}, T_{c}\right]$. The auxiliary Equations (A1), despite their correct formal structure, are therefore not thermodynamically appropriate for justifying the chemical potential according to Equations (A3). Further consideration of the temperature dependence of $u_{v, l}$ shows that the sum $\left(u_{v}+u_{l}\right)$ yields a concavely, but not a convexly curved function, as should be.

The fit function for measured vapor pressure data reads [13] [14]

$$
\ln \left(\frac{p}{p_{c}}\right)=\frac{T_{c}}{T}\left[a_{1} \tau+a_{2} \tau^{1.5}+a_{3} \tau^{3}+a_{4} \tau^{3.5}+a_{5} \tau^{4}+a_{6} \tau^{7.5}\right] \text {, where } \tau \equiv 1-\theta
$$

and $p_{c}=22.064[\mathrm{MPa}]$. The terms $\tau^{1.5}, \tau^{3.5}, \tau^{7.5}$ produce divergent temperature derivatives $\mathrm{d}^{n} p / \mathrm{d} T^{n}$ at $T_{c}$ for $n \geq 2$, whereas all vapor pressure derivatives have finite values. In contrast, the derivatives of the chemical potential $\mathrm{d}^{n} \mu / \mathrm{d} T^{n}$ diverge at $T_{c}$ for $n \geq 2$ and determine the divergence of the heat capacity $C(M, V, T)=M \cdot c(V / M, T)$. In addition, it should be noted here that, if the saturation state is maintained, it is not possible to distinguish between a constant pressure and a constant volume condition. There is thus only one phase-specific heat capacity in the vapor space, $c\left(V_{v} / M_{v}, T\right)$, and one in the liquid space, $c\left(V_{l} / M_{l}, T\right)$, and it holds that

$C(M, V, T)=M_{v} \cdot c\left(V_{v} / M_{v}, T\right)+M_{l} \cdot c\left(V_{l} / M_{l}, T\right)=C\left(M_{v}, V_{v}, T\right)+C\left(M_{l}, V_{l}, T\right)$. 
Numerous thermodynamic deficiencies have been mentioned, viz. violation of the conditions $\alpha(T)<0$ and $\phi(T)>0$ for every temperature $T \subset\left[T_{t}, T_{c}\right]$, the incorrect temperature dependence of $\left(u_{v}+u_{l}\right)$, the proposed vapor pressure fit formula with a divergent term $\mathrm{d}^{2} p / \mathrm{d} T^{2}$ at $T_{c}$ and, finally, tables which list two different phase-specific heat capacities for vapor as well for liquid. From all this it is concluded that the published data [13] [14] are in need of basic correction. 